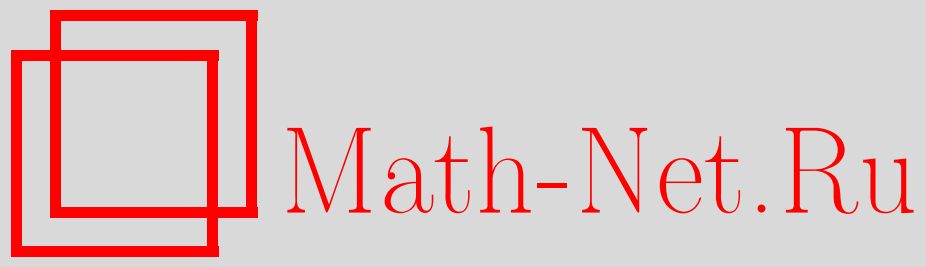

А. М. Вершик, П. Б. Затицкий, Ф. В. Петров, Виртуальная непрерывность измеримых функций многих переменных и теоремы вложения, Функи. анализ и его прил., 2013, том 47, выпуск 3, 1-11

DOI: https://doi.org/10.4213/faa3121

Использование Общероссийского математического портала MathNet.Ru подразумевает, что вы прочитали и согласны с пользовательским соглашением

http://www. mathnet.ru/rus/agreement

Параметры загрузки:

IP : 3.89 .185 .249

26 апреля 2023 г., $17: 50: 17$

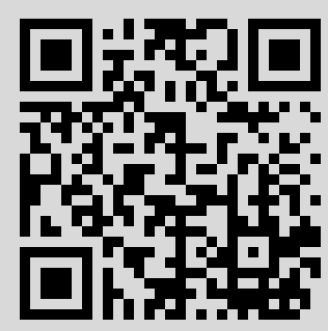


Функционалъный анализ и его приложения

2013, т. 47, вып. 3, с. 1-11

УДК 519.217

\title{
Виртуальная непрерывность измеримых функций многих переменных и теоремы вложения*
}

\author{
(c) 2013. А. М. ВЕРшик, П. Б. ЗАТицКий, Ф. В. ПЕтров
}

\begin{abstract}
Классическая теорема Лузина утверждает, что измеримая функция одной переменной «почти» непрерывна. Для измеримых функций нескольких переменных это уже не так. Поиск правильного аналога этой теоремы приводит к понятию виртуально непрерывных функций нескольких переменных. Это, по-видимому, новое понятие неявно присутствует в утверждениях типа теорем вложения и теорем о следах для пространств Соболева и фактически вскрывает их природу как теорем о виртуальной непрерывности. Особенно полезно это понятие при исследовании и классификации измеримых функций, а также в ряде вопросов теории динамических систем, теории полиморфизмов и бистохастических мер. В этой работе мы напоминаем необходимые определение и свойства допустимых метрик, приводим определение виртуальной непрерывности и формулируем некоторые его приложения, оставляя более подробный анализ для другой статьи.
\end{abstract}

\section{§1. Введение. Допустимые метрики, теорема Лузина}

1.1. Допустимые метрики. Мы рассматриваем стандартное пространство с непрерывной (т. е. неатомической) мерой (пространство Лебега-Рохлина), которое изоморфно отрезку [0, 1] с лебеговой мерой. В работах [4], [8], [11] первого автора предлагалось рассматривать на фиксированном стандартном пространстве с мерой $(X, \mathfrak{A}, \mu)$ различные (допустимые) метрики, в противоположность обычному подходу, при котором фиксируется, наоборот, метрическое пространство и рассматриваются различные борелевские меры на нем. Такой подход полезен и необходим в эргодической теории и в других ситуациях. Согласование структур измеримого и метрического пространств приводит к понятию метрической (или допустимой) тройки:

Определение 1. Метрика (или полуметрика) $\rho$, измеримая как функция двух переменных на стандартном пространстве с мерой $(X, \mathfrak{A}, \mu)$, называется допустимой, если существует измеримое подмножество $X_{0} \subset X$ полной меры $\mu\left(X_{0}\right)=1$, такое, что метрическое (соответственно полуметрическое) пространство $\left(X_{0}, \rho\right)$ сепарабельно.

Стандартное пространство с мерой $(X, \mu)$ и с допустимой (полу)метрикой $\rho$ называется допустимой метрической тройкой или просто допустимой тройкой $(X, \mu, \rho)$.

Свойства допустимых полуметрик и метрик подробно изучены в работах авторов [12], [17]. В частности, там приведен ряд равносильных определений этого понятия.

* Работа выполнена при поддержке гранта РФФИ 11-01-00677-а, гранта Президента России МК-6133.2013.1, а также при поддержке Лаборатории им. П. Л. Чебышева СПбГУ, грант Правительства РФ, дог. 11.G34.31.0026. 
Пользуясь стандартностью пространства, несложно установить следующее

Предложение 1. Если $\rho$ - допустимая метрика на $(X, \mathfrak{A}, \mu)$, то пополненная борелевская сигма-алгебра $\mathfrak{B}=\mathfrak{B}(X, \rho)$ является подалгеброй в $\mathfrak{A}$, мера $\mu$ при этом внутренне регулярна относительно метрики $\rho$, т.е. для любого $A \in \mathfrak{A}$ имеет место равенство

$$
\mu(A)=\sup \{\mu(K): K \subset A, K \text { компактно в метрике } \rho\} .
$$

Таким образом, для любой допустимой метрики $\rho$ исходная мера $\mu$ будет мерой Радона в пространстве $(X, \rho)$.

М. Громов в книге [3] привлек внимание к рассмотрению произвольных метрических троек $(X, \mu, \rho)$, назвав их $m m$-пространствами, и к их классификации, имея в виду классические ситуации (римановы многообразия и др.). Естественно рассматривать в этом контексте допустимые тройки. Определим эквивалентность допустимых троек как эквивалентность с точностью до измеримых изометрий, сохраняющих меру: $(X, \mu, \rho) \sim\left(X^{\prime}, \mu^{\prime}, \rho^{\prime}\right)$, если

существует отображение $T: X \rightarrow X^{\prime}$, такое, что $T \mu=\mu^{\prime}$ и $\rho^{\prime}(T x, T y)=\rho(x, y)$.

Вот основной результат, описывающий эту эквивалентность:

Теорема 1 (Громов [3], Вершик [4]). Рассмотрим отображение

$$
\begin{gathered}
F_{\rho}: X^{\infty} \times X^{\infty} \rightarrow M_{\infty}(\mathbb{R}), \\
F_{\rho}\left(\left\{x_{i}, y_{j}\right\}_{(i, j) \in \mathbb{N} \times \mathbb{N}}\right)=\left\{\rho\left(x_{i}, y_{j}\right)\right\}_{(i, j) \in \mathbb{N} \times \mathbb{N}},
\end{gathered}
$$

и снабдим бесконечное произведение $X^{\infty} \times X^{\infty}$ продакт-мерой $\mu^{\infty} \times \mu^{\infty}$. Тогда образ относительно отображсения $F_{\rho}$ мерь $\mu^{\infty} \times \mu^{\infty}$ есть мера $D_{\rho}$ на пространстве матрич, (или, иными словами, случайная матрица расстояний), называемал матричным распределением метрики $\rho$. Матричное распределение метрики есть полный инвариант класса эквивалентных метрик на пространстве с мерой.

Таким образом,

$$
(X, \mu, \rho) \sim\left(X^{\prime}, \mu^{\prime}, \rho^{\prime}\right) \Longleftrightarrow D_{\rho}=D_{\rho^{\prime}} .
$$

В работе [5] этот результат распространен на так называемые чистые измеримые функции нескольких аргументов.

В теории допустимых метрик полезна следующая

Лемма 1. Пусть $\rho_{1}, \rho_{2}$ - допустимые полуметрики на пространстве Лебега $(X, \mu)$, причем $\rho_{1}-$ метрика. Тогда для любого $\varepsilon>0$ существует измеримое множество $K \subset X$, такое, что $\mu(K)>1-\varepsilon$ и полуметрика $\rho_{2}$ непрерывна на $K \times K$ по метрике $\rho_{1}$ как функиия двух переменных.

В качестве множества $K$ можно взять компакт относительно допустимой метрики $\rho=\rho_{1}+\rho_{2}$, имеющий меру $\mu(K)>1-\varepsilon$.

Из леммы сразу вытекает

Следствие 1. Пусть $\rho_{1}$ и $\rho_{2}-$ две допустимые метрики на пространстве Лебега $(X, \mu)$. Тогда для любого $\varepsilon>0$ существует множество $K \subset X$, такое, что $\mu(K)>1-\varepsilon$ и топологии, задаваемые метриками $\rho_{1} u \rho_{2}$ на $K$, совпадают. 


\section{2. Теорема Лузина об измеримых функциях одного аргумента.} В дальнейшем мы будем говорить об (измеримых) вещественнозначных функциях, хотя большинство результатов остаются верными для отображений в стандартное борелевское пространства, в частности, в произвольное польское пространство. Общеизвестны классические теоремы Егорова и Лузина об измеримых функциях одного аргумента. Сформулируем обобщенную теорему Лузина для произвольной допустимой тройки:

Следствие 2 (теорема Лузина). Пусть $\rho$ - допустимая метрика на пространстве Лебега $(X, \mu)$, a $f$ - измеримое отображсение из $X$ в польское пространство $(M, d)$. Тогда для любого $\varepsilon>0$ существует множество $K \subset X$, такое, что $\mu(K)>1-\varepsilon$ и функиия $f$ на $K$ непрерывна по метрике $\rho$.

Доказательство. Положим $\rho_{1}(x, y)=\rho(x, y)+d(f(x), f(y))$. Ясно, что эта метрика $\rho_{1}$ доставляет тривиальный пример допустимой метрики, относительно которой исходная функция $f$ почти всюду непрерывна. Но по следствию 1 на подходящем множестве $K$ меры $\mu(K)>1-\varepsilon$ из этой непрерывности следует непрерывность функции по $\rho$.

Оказывается, что этот факт не переносится на произвольные функции нескольких аргументов.

\section{§ 2. Виртуальная непрерывность}

2.1. Определение и первые примеры. Для измеримых функций $f(\cdot, \cdot)$ двух переменных на $X \times Y$ аналог теоремы Лузина, т. е. непрерывность на произведении $X^{\prime} \times Y^{\prime} \subset X \times Y$ множеств меры $>1-\varepsilon$ относительно заданной метрики вида $\rho\left[\left(x_{1}, y_{1}\right),\left(x_{2}, y_{2}\right)\right]=\rho_{X}\left(x_{1}, x_{2}\right)+\rho_{Y}\left(y_{1}, y_{2}\right)$, уже выполняется не всегда. Это приводит к следующему понятию, основному в данной работе. (Сумма метрик может быть заменена максимумом или любой другой метрикой, задающей топологию прямого произведения. Чтобы подчеркнуть это обстоятельство, мы обозначаем общую метрику такого класса через $\left.\rho_{X} \times \rho_{Y}.\right)$

Определение 2. Измеримая функция $f(\cdot, \cdot)$ на произведении пространств с мерой $(X, \mu) \times(Y, \nu)$ называется виртуально непреръвной, если для любого $\varepsilon>0$ найдутся множества $X^{\prime} \subset X, Y^{\prime} \subset Y$, меры хотя бы $1-\varepsilon$ каждое, и допустимые полуметрики $\rho_{X}, \rho_{Y}$ на $X^{\prime}, Y^{\prime}$ соответственно, такие, что функция $f$ непрерывна на $\left(X^{\prime} \times Y^{\prime}, \rho_{X} \times \rho_{Y}\right)$. Виртуальная непрерывность функций большего числа аргументов определяется точно так же.

В этом определении существенно, что метрика, относительно которой функция должна быть непрерывной, не произвольна, а учитывает структуру прямого произведения (более общо, наличие фиксированных подалгебр - см. далее). Обратим внимание на то, что не существует универсальной метрики типа произведения, т. е. такой, непрерывность относительно которой равносильна виртуальной непрерывности; это означает, что виртуальная непрерывность не сводится тривиальным образом к обычной непрерывности по какой-то одной метрике.

Существенно и несложно доказать, что всякая допустимая метрика - как измеримая функция двух переменных - виртуально непрерывна. Функция, непрерывная по какой-либо допустимой метрике указанного вида на произведении пространств, разумеется, виртуально непрерывна. Вырожденные функции (или «функции конечного ранга») $f(x, y)=\sum_{i=1}^{n} \varphi_{i}(x) \psi_{i}(y)$, где $\varphi_{i}(\cdot), \psi_{i}(\cdot)$, 
$i=1, \ldots, n,-$ произвольные измеримые функции, также виртуально непрерывны. Достаточно воспользоваться теоремой Лузина для всех функций $\varphi_{i}(\cdot)$, $i=1, \ldots, n$, и $\psi_{i}(\cdot), i=1, \ldots, n$.

Менее тривиальные примеры виртуально непрерывных функций дают, как мы увидим, функции из некоторых классов Соболева, а также ядра ядерных операторов. Для виртуально непрерывных функций можно корректно определять сужения на некоторые множества нулевой меры, а именно, на носители квазибистохастических мер. Об этом см. в следующем разделе.

Простым примером измеримой функции на квадрате $[0,1]^{2}$, не являющейся виртуально непрерывной, может служить характеристическая функция треугольника $\{x \geqslant y\}$. Вообще, в случае функций двух переменных на группе, которые зависят от «частного» двух аргументов, критерий виртуальной непрерывности прост:

Предложение 2. Пусть $G$ - метризуемая компактная группа $u f-$ измеримая по мере Хаара функиия на $G$. Тогда функиия $F(x, y):=f\left(x y^{-1}\right)$ на $G \times G$ виртуально непрерьвна, если и только если функиия $f$ эквивалентна непрерывной.

Подчеркнем, что понятие виртуально непрерывной функции - не топологическое, а чисто теоретико-мерное, т. е. использует только измеримую структуру пространств и меры на них ${ }^{1)}$. По своей природе оно тесно связано с идеей замены метрик на данном пространстве с мерой, о чем шла речь выше. Заметим, что прямой смысл предложения 2 состоит в известном факте, что групповая и теоретико-мерная структуры позволяют восстановить топологию.

2.2. Бистохастические меры и полиморфизмы. С точки зрения теории меры сама по себе функция $k$ аргументов на прямом произведении стандартных пространств с непрерывной мерой есть снова функция на стандартном пространстве с непрерывной мерой - в силу изоморфизма всех таких пространств. Чтобы рассматривать ее именно как функцию $k$ аргументов, следует ввести несколько иную категорию, чем просто категория измеримых пространств.

А именно, рассмотрим пространство с мерой $(\mathscr{X}, \mathfrak{A}, m)$, в сигма-алгебре которого выделено $k$ сигма-подалгебр $\mathfrak{A}_{1}, \ldots, \mathfrak{A}_{k}$. Естественно считать, что эти подалгебры в совокупности порождают все сигма-алгебру.

Связь такой интерпретации с обычной точкой зрения состоит в том, что в пространстве $\mathscr{X}=\prod_{i=1}^{k}\left(X_{i}, \mathfrak{A}_{i}, \mu_{i}\right)$ с мерой $m=\prod \mu_{i}$ подалгебры $\mathfrak{A}_{i}$ отождествляются с подалгебрами алгебры $\mathfrak{A}=\prod \mathfrak{A}_{i}$ путем перемножения на тривиальные подалгебры остальных сомножителей. Иными словами, функции $f\left(x_{1}, \ldots, x_{k}\right)$ на $\mathscr{X}$, измеримые относительно $i$-й сигма-подалгебры $\mathfrak{A}_{i}$, суть функции, зависящие только от одного аргумента $x_{i}(i=1, \ldots, k)$.

Определение 3. Измеримую функцию на пространстве $\mathscr{X}$ с $k$ выделенными подалгебрами, в совокупности порождающими всю сигма-алгебру, будем называть общей измеримой функиией $k$ аргументов.

Рассмотрим меру $\lambda$, заданную на сигма-алгебре $\mathfrak{A}$. Ее можно ограничивать на сигма-подалгебры $\mathfrak{A}_{i}, i=1, \ldots, k$. Будем рассматривать такие меры $\lambda$, что

1) Иначе говоря, это понятие относится к объектам категории пространств с мерой; более традиционный термин для таких понятий в русскоязычной литературе - «метрические понятия», но мы избегаем его из-за нежелательной ассоциации с «метриками»: именно от метрики понятие виртуальной непрерывности не зависит. 
эти ограничения абсолютно непрерывны относительно меры $m$, суженной на $\mathfrak{A}_{i}$. Если ограничения меры $\lambda$ на сигма-алгебры $\mathfrak{A}_{i}$ совпадают с $m, i=1, \ldots, k$, то такая мера называется мультистохастической мерой относительно данных подалгебр (при $k=2$ бистохастической); если же эти ограничения лишь эквивалентны $m$ для $i=1, \ldots, k$, то мы называем меру $\lambda$ почти мультистохастической. Наконец, если $\lambda(U) \leqslant m(U)$ для любого $U \in \mathfrak{A}_{i}, i=1, \ldots, k$, меру $\lambda$ будем называть субмультистохастической.

Разумеется, бистохастическая мера может быть сингулярной относительно произведения мер. Например, в случае прямого произведения отрезков $(X, \mu)=$ $(Y, \nu)=[0,1]$ бистохастической будет мера $\lambda$, сосредоточенная на диагонали $\{x=y\}$ (с плотностью $d \mu(x))$.

Всюду в дальнейшем мы для простоты предполагаем, что $k=2$, т. е. рассматриваем случай двух аргументов, однако никаких серьезных изменений для случая произвольного $k>2$ в наших рассмотрениях и результатах делать не придется. Мы будем рассматривать не только случай независимых аргументов, т. е. не обязательно функции, заданные на прямом произведении двух пространств с непрерывной мерой; большая часть понятий может быть определена для произвольной пары сигма-подалгебр. Но и случай независимых аргументов часто полезнее рассматривать как случай общего положения.

Бистохастические меры на прямом произведении пространств $(X, \mu)$ и $(Y, \nu)$ определяют так называемый полиморфизм пространства $(X, \mu)$ в пространство $(Y, \nu)$ (см. [9]), т. е. «многозначное отображение» с инвариантной мерой. Особенно важен случай отождествленных аргументов: $(X, \mathfrak{A}, \mu)=(Y, \mathfrak{B}, \nu)$; тогда полиморфизм обобщает понятие автоморфизма пространства с мерой. Почти бистохастическая мера определяет полиморфизм с квазиинвариантной мерой. Бистохастическая или почти бистохастическая мера $\lambda$ определяет также билинейную (в общем случае $k$-линейную) форму $(f(x), g(y)) \rightarrow \int f(x) g(y) d \lambda(x, y)$, которой соответствует так называемый марковский (соответственно квазимарковский) оператор в соответствующих функциональных пространствах. Заметим лишь, что этот оператор $U_{\lambda}$ есть сжатие, т. е. его норма не превышает единицы, он оставляет инвариантным конус неотрицательных функций. В случае бистохастической меры этот оператор, как и его сопряженный, оставляет неподвижными константы: $U_{\lambda} 1=1$. О многочисленных связях теории полиморфизмов (марковские операторы, джойнинги, бирасслоения, соответствия, меры Юнга, каплинги и т.д.) см. в [9], [10], [7]. Бистохастические меры играют ключевую роль в бурно развивающейся теории непрерывных графов [15].

2.3. Дальнейшие свойства виртуально непрерывных функций. Прежде всего, виртуально непрерывные функции автоматически обладают более сильными свойствами, чем это требуется в определении. С одной стороны, можно добиться того, чтобы множества $X^{\prime}, Y^{\prime}$ из определения имели полную меру:

Теорема 2. Пусть функиия $f(\cdot, \cdot)$ виртуально непрерьвна. Тогда найдутся множества $X^{\prime} \subset X, Y^{\prime} \subset Y$, полной меры каждое, и допустимые полуметрики $\rho_{X}, \rho_{Y}$ на $X^{\prime}, Y^{\prime}$ соответственно, такие, что функиия $f$ непреръвна нa $\left(X^{\prime} \times Y^{\prime}, \rho_{X} \times \rho_{Y}\right)$.

С другой стороны, метрики можно считать наперед заданными: 
Теорема 3. Пусть функиия $f(\cdot, \cdot)$ виртуально непрерьвна. Тогда для любого $\varepsilon>0$ и любых допустимых метрик $\rho_{X}, \rho_{Y}$ на $X, Y$ найдутся множества $X^{\prime} \subset X, Y^{\prime} \subset Y$, меры хотя бы $1-\varepsilon$ каждое, такие, что функиия $f$ непрерывна на $\left(X^{\prime} \times Y^{\prime}, \rho_{X} \times \rho_{Y}\right)$.

Функцию $f$ двух переменных на $X \times Y$ полезно рассматривать как отображение из $X$ в пространство функций на $Y$ (т. е. $\left.f(x, y) \equiv f_{x}(y)\right)$. Более подробно о том, как использовать это для классификации измеримых функций, см. в [16]. Виртуальная непрерывность описывается в этих терминах следующим равносильным определением:

Теорема 4. Виртуальная непрерывность функиии $f(\cdot, \cdot)$ равносильна ее следующему свойству: для любого $\varepsilon>0$ найдутся множества $X^{\prime} \subset X, Y^{\prime} \subset Y$, меры хотя бы $1-\varepsilon$ каждое, такие, что множество функций вида $f_{x}(\cdot)$ на $Y^{\prime}$ (переменная $x$ пробегает $X^{\prime}$ ) образует вполне ограниченное (предкомпактное) семейство в $L_{\infty}\left(Y^{\prime}\right)$.

Из этого определения следует важный вывод: непрерывность функции по одной переменной влечет за собой виртуальную непрерывность.

Лемма 2. Пусть $(X, \mu),(Y, \nu)$ - стандартные непрерывные вероятностные пространства и $\rho_{Y}$ - допустимая метрика на множестве $Y^{\prime} \subset Y$ полной меры. Пусть измеримая функиия $f: X \times Y \rightarrow \mathbb{R}$ такова, что при $\mu$-почти всех $x \in X$ функиия $f(x, \cdot)$ непрерывна на $\left(Y^{\prime}, \rho_{Y}\right)$. Тогда функция $f$ виртуально непрерывна.

Из теоремы 2 немедленно получаем, что верно и обратное: для виртуально непрерывной функции $f$ найдется соответствующая метрика $\rho_{Y}$. Таким образом, условие леммы 2 (непрерывность по подходящей метрике по $y$ при почти всех фиксированных $x$ ) равносильно виртуальной непрерывности функции.

Замечательно, что в этом определении роль пространств $X$ и $Y$ (т. е. аргументов функции) различна; тем не менее, апостериори это свойство симметрично относительно замены порядка аргументов. В этом также сказывается нетривиальность понятия виртуальной непрерывности.

Измеримые функции $f(\cdot, \cdot)$, как мы видели, классифицируются матричными распределениями, т.е. мерами на пространстве бесконечных матриц $\left(a_{i j}\right)_{i, j=1}^{\infty}$, индуцируемыми отображением $f \rightarrow\left(a_{i, j}=f\left(x_{i}, y_{j}\right)\right)$; точки $x_{i}$ в $X$ и $y_{i}$ в $Y, i=1,2, \ldots$, выбираются независимо. В этих терминах можно охарактеризовать свойство виртуальной непрерывности:

Теорема 5. Пусть точки $x_{1}, x_{2}, \ldots$ выбираются случайно и независимо в $X$, а точки $y_{1}, y_{2}, \ldots$ - случайно и независимо в $Y$. Виртуальная непрерывность функции $f(x, y)$ равносильна тому, что для любого $\varepsilon>0$ найдется натуральное $N$, при котором вероятность следующего события равна 1 :

найдутся такие разбиения натурального ряда $\{1,2, \ldots\}=\bigsqcup_{i=0}^{N} A_{i}=\bigsqcup_{i=0}^{N} B_{i}$, что верхняя плотность множества $A_{0} \cup B_{0}$ менъше $\varepsilon$ (m.е. $\lim \sup \mid\left(A_{0} \cup B_{0}\right) \cap$ $[1, n] \mid / n<\varepsilon) u\left|f\left(x_{s}, y_{t}\right)-f\left(x_{r}, y_{p}\right)\right|<\varepsilon$ nрu $i, j>0, s, r \in A_{i}, p, t \in B_{j}$.

Из приведенных характеристик виртуальной непрерывности легко вывести, что в пространстве всех измеримых функций двух переменных (с топологией сходимости по мере) совокупность виртуально непрерывных функций образует нигде не плотное множество.

2.4. Толщина. Рассмотрим пространство $X \times Y$ с мерой $\mu \times \nu$; в сигмаалгебре его измеримых множеств естественно выделено две сигма-подалгебры, 
определяемые проекциями на $X$ и $Y$. Назовем толщиной $\operatorname{th}(Z)$ измеримого множества $Z \subset X \times Y$ точную нижнюю грань величины $\mu\left(X_{1}\right)+\nu\left(Y_{1}\right)$ по всем парам измеримых подмножеств $X_{1} \subset X, Y_{1} \subset Y$, таких, что

$$
(\mu \times \nu)\left(Z \backslash\left(X_{1} \times Y \cup X \times Y_{1}\right)\right)=0
$$

Множества $X_{1} \times Y, X \times Y_{1}$ суть в точности множества из выделенных сигмаподалгебр, что позволяет определить обобщенную толщину для других выделенных сигма-алгебр в стандартном пространстве. Отметим непосредственно вытекающие из определения свойства введенного понятия:

- толщина множества всегда не превосходит 1 и равна 0 в точности для множеств меры 0 ;

- толщина подмножества не превосходит толщины множества;

- толщина множества не меньше его меры;

- толщина конечного или счетного объединения множеств не превосходит суммы толщин.

Чуть менее очевидна

Лемма 3. Если $\operatorname{th}(Z)=0$, то в (1) можно выбрать множества $X_{1}, Y_{1}$ меръь 0 .

Выражение «сколь угодно тощее множество» будет означать «множество сколь угодно малой толщины». В этих терминах лемма 3 может быть переформулирована так: если множество может быть покрыто сколь угодно тощим множеством, то его дополнение содержит mod 0 произведение множеств полной меры.

Равносильное и в ряде ситуаций более удобное для применений определение толщины дает следующая

Лемма 4. Для любого множества $Z \subset X \times Y$ рассмотрим пары измеримых функиий $f: X \rightarrow[0,1], g: Y \rightarrow[0,1], \operatorname{makux,~что~} f(x)+g(y) \geqslant \chi_{Z}(x, y)$ для $\mu \times \nu$-почти всех пар $(x, y)$. Тогда толщина множества $Z$ есть точная нижняя грань суммы интегралов $\int_{X} f d \mu u \int_{Y} g d \nu$.

Применяя эту лемму и переходя к слабо сходящимся подпоследовательностям функций, можно установить «непрерывность толщины снизу»:

Лемма 5. Пусть $\left\{Z_{n}\right\}$ - возрастающая последовательность измеримых множеств, $Z=\bigcup_{n} Z_{n}$. Тогда $\operatorname{th}(Z)=\lim \operatorname{th}\left(Z_{n}\right)$.

Отметим, что непрерывность толщины сверху не имеет места: все множества $\{(x, y): 0<|x-y|<1 / n\} \subset[0,1]^{2}$ имеют толщину 1 , но их пересечение пусто.

Определим сходимость функций «по толщине» по аналогии со сходимостью по мере. Это сходимость в метризуемой топологии, задаваемой таким расстоянием:

Определение 4. Зададим расстояние $\tau(f(x, y), g(x, y))$ между двумя произвольными измеримыми функциями двух переменных как точную нижнюю грань тех $\varepsilon>0$, для которых $\operatorname{th}\{(x, y):|f(x, y)-g(x, y)|>\varepsilon\} \leqslant \varepsilon$.

Нетрудно доказать, что это есть метрика в пространстве непрерывных функций; назовем ее $\tau$-метрикой. Сходимость по введенной $\tau$-метрике влечет за собой сходимость по мере (но не наоборот). 
Пусть $\xi_{X}: X=\bigsqcup_{i=1}^{n} X_{i}$ и $\xi_{Y}: Y=\bigsqcup_{i=1}^{m} Y_{i}-$ конечные разбиения пространств $X$ и $Y$ соответственно на измеримые подмножества положительной меры. Функции, постоянные mod 0 на каждом из прямых произведений $X_{i} \times Y_{j}$, будем называть ступенчатыми. Конечные линейные комбинации $\sum_{i=1}^{N} a_{i}(x) b_{i}(y)$ будем называть функииями конечного ранга. Множество измеримых функций полно по $\tau$-метрике.

Связь с виртуальной непрерывностью дает следующая

Теорема 6. Замыкания по $\tau$-метрике множества ступенчатых функций (или множества функций конечного ранга) есть в точности множество виртуально непрерывных функиий.

Здесь тоже виден чисто теоретико-мерный метрический характер понятия виртуальной непрерывности, а также возможность определения этого понятия для произвольной пары сигма-подалгебр. Относительно близких понятий см. также [13].

2.5. Норма в пространстве виртуально непрерывных функций. Мы определили сходимость по $\tau$-метрике, являющуюся для виртуально непрерывных функций аналогом сходимости по мере. Можно ввести аналоги известных банаховых пространств измеримых функций.

Измеримую функцию $h(\cdot, \cdot)$ на пространстве $(X \times Y, \mu \times \nu)$ назовем суббистохастической, если мера с плотностью $|h(\cdot, \cdot)|$ относительно продакт-меры на $X \times Y$ является суббистохастической. Множество суббистохастических функций обозначим через $\mathscr{S}$.

Определим конечную или бесконечную норму измеримой функции $f(\cdot, \cdot)$ равенством

$$
\|f\|:=\inf \left\{\int_{X}|a(x)| d \mu(x)+\int_{Y}|b(y)| d \nu(y):|f(x, y)| \leqslant a(x)+b(y) \text { п.в. }\right\} .
$$

Следующая теорема есть аналог известной теоремы двойственности Канторовича [1] в транспортной задаче - а именно, двойственности между пространством мер с метрикой Канторовича и пространством функций Липшица (см. также [18]).

Теорема 7. Имеет место равенство

$$
\|f\|=\sup \left\{\int_{X \times Y}|f(x, y)| h(x, y) d x d y: h \in \mathscr{S}\right\} .
$$

Совпадение инфимума и супремума и есть утверждение о наличии двойственности в задаче бесконечномерного линейного программирования. Однако в нашем случае доказательство требует более тонких соображений, чем в случае задачи Монжа-Канторовича, поскольку мы рассматриваем пространство типа $L^{1}$, в котором конус неотрицательных функций (в отличие от пространства непрерывных функций, используемого в транспортной задаче) не имеет внутренних точек. Последнее не позволяет напрямую применить стандартные теоремы отделимости.

Теорема 8. Замыкание множества ступенчатых функиий в рассматриваемой норме состоит из всех виртуально непрерывных функиий, у которых эта норма конечна (в частности, любая ограниченная виртуально непрерывная функция лежит в этом замыкании). 
Обозначим это пространство через $V C^{1}$. Оно является аналогом пространства $L^{1}$ для виртуально непрерывных функций и является предсопряженным к пространству полиморфизмов с ограниченными плотностями проекций.

Теорема 9. Сопряэенным $к$ пространству $V C^{1}$ является пространство зарядов $\eta$ на $X \times Y$ с конечной нормой

$$
\|\eta\|_{\text {me }}=\max \left\{\left\|\frac{\partial P_{*}^{x}|\eta|}{\partial \mu}\right\|_{L^{\infty}(X, \mu)}, \|\left.\frac{\partial P_{*}^{y}|\eta|}{\partial \nu}\right|_{L^{\infty}(Y, \nu)}\right\},
$$

где $P^{x}$ и $P^{y}$ - проекиии на $X$ и $Y$ соответственно, а $|\eta|$ - вариация заряда.

Следствие 3. Для виртуально непрерывных функиий из пространства $V C^{1}$ (в частности, для ограниченных виртуально непрерывных функиий) корректно определен интеграл не только по множсествам положстельной меры, как для произвольных суммируемых функиии, но и по бистохастическим (сингулярным) мерам, например, по мере Лебега на диагонали $\{x=y\} \subset[0,1]^{2}$ или по мере, сосредоточенной на графике преобразования с квазиинвариантой мерой. Таким образом, виртуально непрерывные бункиии имеют «след (ограничение) на диагонали» в смысле теорем о следах.

\section{§3. Приложения: теоремы вложения, теоремы о следах и ограничение метрик}

Укажем на некоторые приложения понятия виртуальной непрерывности.

\section{1. Пространства Соболева и теоремы о следах.}

Теорема 10. Пусть $\Omega_{1}, \Omega_{2}$ - области размерностей $d_{1}, d_{2}$ соответственно и $p l>d_{2}$ либо $p=1, l=d_{2}$. Тогда функции из $W_{p}^{l}\left(\Omega_{1} \times \Omega_{2}\right)$ (т.е. функиии, l-е обобщенные производные которых суммируемы со степенъю р) виртуально непрерывны как функции двух переменных $x \in \Omega_{1}, y \in \Omega_{2}$. Вложение пространства $W_{p}^{l}\left(\Omega_{1} \times \Omega_{2}\right)$ в $V C^{1}\left(\Omega_{1}, K\right)$ непрерывно для любого компакта $K$ в области $\Omega_{2}$.

Доказательство. Согласно теореме вложения в пространство непрерывных функций (см., например, [2], [6]), для функций $h(y) \in W_{p}^{l}\left(\Omega_{2}\right)$ имеет место оценка

$$
\|h\|_{C(K)} \leqslant c\left(\Omega_{2}, K\right)\|h\|_{W_{p}^{l}\left(\Omega_{2}\right)} .
$$

Пусть $f(x, y) \in W_{p}^{l}\left(\Omega_{1} \times \Omega_{2}\right)$ - гладкая функция. Положим

$$
a(x):=\|f(x, \cdot)\|_{W_{p}^{l}\left(\Omega_{2}\right)} .
$$

Тогда по теореме Фубини $a \in L^{1}\left(\Omega_{1}\right)$ и

$$
\int|a| \leqslant c\left(\Omega_{1}, \Omega_{2}\right)\|f(x, y)\|_{W_{p}^{l}\left(\Omega_{1} \times \Omega_{2}\right)} .
$$

На $\Omega_{1} \times K$ имеем

$$
|f(x, y)| \leqslant\|f(x, \cdot)\|_{C(K)} \leqslant c\left(\Omega_{2}, K\right) a(x) .
$$

Резюмируя, получаем, что

$$
\|f\|_{V C^{1}\left(\Omega_{1}, K\right)} \leqslant c\left(\Omega_{1}, \Omega_{2}, K\right)\|f\|_{W_{p}^{l}\left(\Omega_{1} \times \Omega_{2}\right)} .
$$


Любая функция из класса $W_{p}^{l}\left(\Omega_{1} \times \Omega_{2}\right)$ есть предел последовательности гладких функций, который в силу (2) является и пределом в смысле нормы пространства $V C^{1}$ и, следовательно, виртуально непрерывной функцией.

Заметим, что та метрика, в которой эта виртуально непрерывная функция является непрерывной, может совсем не совпадать и даже не быть эквивалентной метрике, присутствующей в исходной постановке задаче (например, евклидовой метрике). Таким образом, в условиях указанной теоремы о вложении мы получаем возможность интегрировать функции по любой квазибистохастической мере, что обобщает обычные теоремы о следах на подмногообразиях.

3.2. Ядерные операторы в гильбертовом пространстве. Хорошо известно, что пространство ядерных операторов в гильбертовом пространстве $L^{2}$ есть проективное тензорное произведение гильбертовых пространств. Их ядра суть измеримые функции двух переменных, прямое описание которых трудно обозримо. Следующая теорема утверждает, что ядра ядерных интегральных операторов как функции двух переменных виртуально непрерывны. Заметим, что ядра операторов Гильберта-Шмидта, вообще говоря, не являются виртуально непрерывными.

Теорема 11. Пусть $(X, \mu),(Y, \nu)$ - стандартные вероятностные пространства. Пространство ядер ядерных операторов из $L^{2}(X)$ в $L^{2}(Y)$ (с нормой Шаттена-фбон Неймана) непрерьвно вкладывается в $V C^{1}$.

Отсюда следует, что эти ядра можно интегрировать не только по диагонали, когда $X=Y$, что хорошо известно, но и по бистохастическим мерам. Однако пространство $V C^{1}$ не исчерпывается этими ядрами. Следует также помнить, что если рассматривать пространство $V C^{1}$ как пространство ядер операторов в подходящих пространствах $L^{p}$, то оно не является инвариантным относительно сопряжения унитарными операторами, как это имеет место для пространств Шаттена-фон Неймана и, в частности, пространства ядерных операторов. Действительно, в определении пространства $V C^{1}$ существенно участвуют известные сигма-подалгебры, которые не обладают нужной инвариантностью. См. работу [14], где рассматривается близкий вопрос.

3.3. Ограничение метрик. Следующая задача была одним из источников проблематики данной работы. Пусть $(X, \mu)$ - стандартное пространство с непрерывной мерой. Предположим, что $\rho$ - допустимая метрика и $\xi-$ измеримое разбиение пространства $(X, \mu)$, элементы которого имеют меру нуль, например, $\xi$ - разбиение на линии уровня функции, не постоянной ни на каких множествах положительной меры. Можно ли корректно ограничить метрику (как функцию двух переменных) на элементы разбиения?

Ответ на этот вопрос не столь очевиден, так как метрика здесь априори всего лишь измеримая функция. Однако, как утверждалось выше, всякая допустимая метрика виртуально непрерывна, и потому для получения положительного ответа достаточно указать ту бистохастическую меру, на носитель которой следует ограничить метрику. Для простоты будем считать, что $X=[0,1]^{2}, \mu$ - лебегова мера на квадрате, а разбиение $\xi$ - разбиение единичного квадрата на вертикальные отрезки. Тогда речь идет об ограничении виртуально непрерывной функции, заданной на $X^{2}=[0,1]^{4}$, на трехмерное подмногообразие: $\left\{\left(x_{1}, x_{2}, x_{3}, x_{4}\right): x_{1}=x_{3}\right\}$. Легко видеть, что это подмногообразие, снабженное трехмерной лебеговой мерой, определяет бистохастическую меру на $X \times X$. 
Благодарности. Авторы признательны Л. Ловасу, приславшему свою недавно вышедшую монографию [15], в которой, в частности, обсуждаются некоторые близкие вопросы, и А. Логунову, обратившему наше внимание на возможность двойственного определения понятия толщины.

\section{ЛитеРАТУРА}

[1] Л. Канторович, О транспортировке масс, ДАН СССР, 37:7-8 (1942), 227-229.

[2] В. Г. Мазья, Пространства Соболева, Изд-во ЛГУ, Л., 1985.

[3] M. Gromov, Metric Structure for Riemannian and Non-Riemannian Spaces, Birkhauser, Boston, 1998.

[4] А. Вершик, Универсальное пространство Урысона, метрические тройки Громова и случайные метрики на натуральном ряде, УМН, 53:5 (1998), 57-64.

[5] А. Вершик, Классификачия измеримых функиий нескольких аргументов и инвариантно распределенные случайные матрицы, Функц. анализ и его прил., $\mathbf{3 6 : 2}$ (2002), 12-28.

[6] R. A. Adams, J. J. F. Fournier, Sobolev Spaces, Pure and Applied Mathematics, vol. 140, Elsevier/Academic Press, Amsterdam, 2003.

[7] A. Vershik, Random and universal metric spaces, in: Dynamics and Randomness II, Kluwer Academic Publ., Dordrecht, 2004, 199-228.

[8] А. М. Вершик, Случайные метрические пространтва и универсальность, УМН, 59:2(356) (2004), 65-104.

[9] A. Vershik, Polymorphisms, Markov processes, and quasi-similarity, Discrete Contin. Dyn. Syst., 13:5 (2005), 1305-1324.

[10] A. Vershik, Как выцлядит типичный марковский оператор?, Алгебра и анализ, 17:5 (2005), 91-104.

[11] A. Vershik, Dynamic of metrics in measure spaces and their asymptotic invariants, Markov Processes Related Fields, 16:1 (2010), 169-185.

[12] П. Б. Затицкий, Ф. В. Петров, Об исправлении метрик, Зап. научн. сем. ПОМИ, 390 (2011), 201-209.

[13] K-T. Sturm, The space of spaces:curvature bounds and gradient flows on the space of metric measure space, Preprint.

[14] M. Denker, M. Gordin, Limit theorem for von Mises statistics of a measure preserving transformations, http://arxiv.org/abs/1109.0635v2.

[15] L. Lovasz, Large Networks and Graph Limits, Colloquium Publications, vol. 60, Amer. Math. Soc., Providence, RI, 2012.

[16] А. Вершик, О классификации измеримых функиий нескольких переменных, Зап. научн. сем. ПОМИ, 403 (2012), 35-57.

[17] F. Petrov, A. Vershik, P. Zatitskiy, Geometry and dynamics of admissible metrics in measure spaces, Central Europ. J. Math., 11:3 (2013), 379-400.

[18] A. Vershik, Long History of Monge-Kantorovich transportation problem, Mathem. Intellegencer, 35:4 (2013).

ПОМИ им. В. А. Стеклова РАН и

Санкт-Петербургский государственный университет

Поступило в редакцию

e-mail: avershik@gmail.com

ПОМИ им. В. А. Стеклова РАН и

Санкт-Петербургский государственный университет,

Исследовательская лаборатория им. П. Л. Чебышева

e-mail: paxa239@yandex.ru

ПОМИ им. В. А. Стеклова РАН и

Санкт-Петербургский государственный университет

e-mail: fedyapetrov@gmail.com 